\title{
TU/e EmonOWEN

\section{Effects of migrating cell-induced matrix reorganization on 3D cancer cell migration}

\section{Citation for published version (APA):}

Sun, W., Kurniawan, N. A., Kumar, A. P., Rajagopalan, R., \& Lim, C. T. (2014). Effects of migrating cell-induced matrix reorganization on 3D cancer cell migration. Cellular and Molecular Bioengineering, 7(2), 205-217. https://doi.org/10.1007/s12195-014-0324-0

DOI:

10.1007/s12195-014-0324-0

Document status and date:

Published: 01/06/2014

\section{Document Version:}

Publisher's PDF, also known as Version of Record (includes final page, issue and volume numbers)

\section{Please check the document version of this publication:}

- A submitted manuscript is the version of the article upon submission and before peer-review. There can be important differences between the submitted version and the official published version of record. People interested in the research are advised to contact the author for the final version of the publication, or visit the $\mathrm{DOI}$ to the publisher's website.

- The final author version and the galley proof are versions of the publication after peer review.

- The final published version features the final layout of the paper including the volume, issue and page numbers.

Link to publication

\section{General rights}

Copyright and moral rights for the publications made accessible in the public portal are retained by the authors and/or other copyright owners and it is a condition of accessing publications that users recognise and abide by the legal requirements associated with these rights.

- Users may download and print one copy of any publication from the public portal for the purpose of private study or research.

- You may not further distribute the material or use it for any profit-making activity or commercial gain

- You may freely distribute the URL identifying the publication in the public portal.

If the publication is distributed under the terms of Article 25fa of the Dutch Copyright Act, indicated by the "Taverne" license above, please follow below link for the End User Agreement:

www.tue.nl/taverne

Take down policy

If you believe that this document breaches copyright please contact us at:

openaccess@tue.nl

providing details and we will investigate your claim. 


\title{
Effects of Migrating Cell-Induced Matrix Reorganization on 3D Cancer Cell Migration
}

\author{
Wei Sun, ${ }^{1}$ Nicholas Agung Kurniawan, ${ }^{1,7}$ Alan Prem Kumar, ${ }^{2}$ Raj Rajagopalan, ${ }^{1,3,8}$ \\ and ChweE TeCK Lim ${ }^{1,4,5,6}$
}

${ }^{1}$ NUS Graduate School for Integrative Sciences and Engineering, Singapore 117456, Singapore; ${ }^{2}$ Cancer Science Institute, National University of Singapore, Singapore 117599, Singapore; ${ }^{3}$ Department of Chemical and Biomolecular Engineering, National University of Singapore, Singapore 117411, Singapore; ${ }^{4}$ Department of Mechanical Engineering, National University of Singapore, Singapore 117411, Singapore; ${ }^{5}$ Department of Bioengineering, National University of Singapore, Singapore 117576, Singapore; ${ }^{6}$ Mechanobiology Institute, National University of Singapore, Singapore 117411, Singapore; ${ }^{7}$ FOM Institute AMOLF, Science Park 102, 1098XG Amsterdam, The Netherlands; and ${ }^{8}$ Skolkovo Institute of Science and Technology, Moscow, The Russian Federation

(Received 11 September 2013; accepted 11 February 2014; published online 19 February 2014)

Associate Editor Michael R. King oversaw the review of this article.

\begin{abstract}
The migration of cells is fundamental to a number of physiological/pathological processes, ranging from embryonic development, tissue regeneration to cancer metastasis. Current research on cell migration is largely based on simplified in vitro models that assume a homogeneous microenvironment and overlook the modification of extracellular matrix (ECM) by the cells. To address this shortcoming, we developed a nested threedimensional (3D) collagen hydrogel model mimicking the connective tissue confronted by highly malignant breast cancer cells, MDA-MB-231. Strikingly, our findings revealed two distinct cell migration patterns: a rapid and directionally persistent collective migration of the leader cells and a more randomized migration in the regions that have previously been significantly modified by cells. The cell-induced modifications, which typically include clustering and alignment of fibers, effectively segmented the matrix into smaller sub-regions. Our results suggest that in an elastic $3 \mathrm{D}$ matrix, the presence of adjacent cells that have modified the matrix may in fact become physical hurdle to a migrating cell. Furthermore, our study emphasizes the need for a micromechanical understanding in the context of cancer invasion that allows for cell-induced modification of ECM and a heterogeneous cell migration.
\end{abstract}

Keywords-Cancer invasion, 3D extracellular matrix, Collective cell migration, Matrix remodeling, Cell contraction.

\section{INTRODUCTION}

Metastasis refers to the spreading of cancer cells from the original tumor sites to one or more new locations in the body, followed by secondary cancerous growth. ${ }^{19} \mathrm{It}$

Address correspondence to Nicholas Agung Kurniawan, FOM Institute AMOLF, Science Park 102, 1098XG Amsterdam, The Netherlands. Electronic mail: kurniawan@amolf.nl is responsible for $90 \%$ of the deaths caused by cancer. ${ }^{45}$ Metastasis starts with the infiltration of loose stromal tissue by migrating tumor cells, leading to local invasion. ${ }^{21}$ Each step involves an intimate physico-chemical relationship between the tumor cells and the surrounding environment. ${ }^{47}$ The complex series of migration stages in vivo has presented a challenging in vitro problem, especially in order to understand the cancer cell migratory behavior, which is crucial both in the fundamental research of cell migration and in the development of effective therapeutics for cancer.

Two-dimensional (2D) cell culture techniques have been widely used in cell migration assays. Lately, however, three-dimensional (3D) hydrogel matrixbased cell culture systems have gained popularity as in vitro models for studying pathological processes, such as cancer cell invasion, primarily for the improved recapitulation of extracellular environment. ${ }^{38,41,48}$ As an improvement over traditional 2D models for studying cancer cell migration, a close-to-3D model has recently been developed, which allowed for visualization of the details of matrix degradation during the development of cells' invadopodia. ${ }^{52}$ For genuine 3D cell culture, hydrogel models have been typically built with hydrated collagen, which is a major protein component of connective tissues. ${ }^{34}$ Such models have been increasingly adopted in 3D cell culture systems and cell migration assays, ${ }^{17,33,50}$ as these $3 \mathrm{D}$ collagen matrix models provide a more physiologically relevant and controlled environments for migrating cells, ${ }^{53}$ and also allow for cell-induced remodeling to the matrix through extracellular matrix (ECM) protein synthesis, proteolytic degradation and matrix contraction. 
Among these factors, the physical remodeling of 3D ECM can change the microstructure of the matrix ${ }^{27}$ and result in multiple implications on cell migration. Firstly, mechanical contraction of ECM has been shown to facilitate cell migration. For example, the contraction of collagen matrix is closely associated with the invasive migration of wild-type and transformed fibroblasts in 3D gel system. ${ }^{30,33}$ Collagen fibrils are pulled by migrating cells through contraction, ${ }^{24}$ and the local deformation of fibrillar collagen depend on the cytoskeleton dynamics, ${ }^{36}$ focal adhesion, ${ }^{37}$ and cell contractility. ${ }^{46}$ Secondly, physical remodeling may induce long term and significant changes to the matrix. ${ }^{28}$ For example, a recent study suggests that malignant human breast cancer cells are able to reorient the fibrillar collagen to be perpendicular to the tumor boundary, and this in turn enhances cell invasion into the collagen matrix. ${ }^{39}$ Tumor stromal cells, such as fibroblasts, have been found to prime the tumor microenvironment in favor of tumor invasion. It is also noteworthy that tissue remodeling is highly force-dependent, ${ }^{31}$ both in vivo and in vitro. ${ }^{16}$

In contrast to these detailed findings on the single cell level, how matrix reorganization influences the cell population as a whole remains unclear. However, it has already been recognized that collective cell migration contributes greatly to cancer metastasis. ${ }^{7,11}$ Recent studies on collective cell migration have led to the development of a number of in vitro models, which include seeding cells on 2D or in 3D uniform environment, subjecting cells to barriers ${ }^{8,10}$ and immobilizing ECM proteins to form patterns on a 2D substrate. ${ }^{22,32}$ These models have particularly focused on the relation between cell migration and the predetermined ECM conditions (see recent review by Schedin and Keely ${ }^{43}$ ), but the feedback effects of cell modifications to the ECM on cell migration has not been analyzed.

In this work, we examine whether cell-induced matrix reorganization on the micro-scale alters contractile cell movement, in the context of cancer cell invasive migration in the stromal tissue. We chose a highly metastatic breast cancer cell line, MDA-MB-231, as a model for metastatic cell population, and we accordingly developed a cell migration assay with nested 3D collagen to mimic the connective tissue around an invading tumor. Based on this system, we identified spatially and temporally heterogeneous cell migration patterns. In particular, as compared with cells on the migration front, the cell movement speed and directionality were lower in the wake of the migration front. The reduction of cell motility was associated with higher cell population density in collagen, which may result in enhanced segmentation of the matrix, i.e., a physical partitioning of local fiber matrix due to remodeling by cells. The finding that cell migration is achieved more efficiently in the matrix areas that have not yet been occupied or reorganized may provide one physical explanation for cancer cells' proclivity to spread into a cell-free tissue.

\section{MATERIALS AND METHODS}

\section{Cell Culture}

Nutragen ${ }^{\mathrm{TM}}$ collagen solution was produced by Inamed Biomaterials (Fremont, CA), mainly containing bovine collagen-I $(97 \%)$ and collagen-III $(3 \%)$. The collagen used is pepsin-treated and forms non-crosslinked networks, allowing for MMP-independent cell invasion. ${ }^{35}$ Malignant human breast cancer cell line cells (MDA-MB-231; ATCC) were maintained in Dulbecco's modified Eagle's medium (DMEM, Mediatech), supplemented with $10 \%$ FBS and $1 \%$ penicillin/streptomycin, under $37^{\circ} \mathrm{C}$ humidified atmosphere with $5 \%$ $\mathrm{CO}_{2}$. Benign human breast tumor cells (MCF-10A; ATCC) were used as a control.

\section{D NESTED-GELS CELL INVASION ASSAY}

The collagen precursor solution was prepared by neutralizing (to $\mathrm{pH}$ 7.4) the acidic stock solution with $\mathrm{NaOH}$ and mixing with $10 \times$ PBS, according to the protocol provided by the manufacturer (Inamed Biomaterials, 2006). The cultured cells were harvested through trypsin/EDTA treatment and mixed with collagen solution on ice. Finally $1 \mathrm{~mL}$ of the solution contained 1.5-2 million cells and $2.5 \mathrm{mg}$ collagen. $20 \mu \mathrm{L}$ of the above liquid mixture were transferred to the center of the $1.3 \mathrm{~cm}$ round well of a glass-bottom dish (MatTek, Ashland, MA). 30-60 min incubation at $37{ }^{\circ} \mathrm{C}$ humid chamber led to the self-assembly of a semi-spherical cell-seeded collagen gel ("inner gel"). Next, $180 \mu \mathrm{L}$ of 1.5 or $2.5 \mathrm{mg} / \mathrm{mL}$ collagen solution (diluted in DMEM) was added to cover the small gel and polymerized under the same condition to form a dome-shaped "outer gel". We found negligible difference in using the two outer gel collagen concentrations (data not shown), so in this study we do not differentiate the results obtained from the two conditions. $2 \mathrm{~mL}$ phenol-red-free DMEM (GIBCO, Life Technologies) containing 10\% FBS and 1\% penicillin/ streptomycin was supplied to the gel in the glass-bottom dish, and the medium was changed every 3 days throughout the 2-3 weeks of the experiment. For contractility inhibition studies, $20 \mu \mathrm{M}$ of ROCK inhibitor Y-27632 (Santa Cruz Biotechnology, Inc., Santa Cruz, CA) was added to the complete culture medium from Day 6 onwards. 


\section{Imaging of Cells and Collagen Matrix}

Time-lapse microscopy was carried out in a temperature-controlled chamber keeping cells under $37{ }^{\circ} \mathrm{C}$ and $5 \% \mathrm{CO}_{2}$ atmosphere, starting from 4 days after cell seeding. Utilizing Nikon TE-2000 inverted microscope (Nikon, Tokyo, Japan), live images were obtained through light microscopy using differential interference contrast microscopy (DIC) and confocal laser scanning microscopy (CLSM) every $10 \mathrm{~min}$, which is comparable to the cytoskeleton turnover rate. For confocal fluorescence imaging, cells were labeled in situ with $10 \mu \mathrm{M}$ fluorescence dyes, i.e., CellTracker CMFDA and CMTMR (Molecular Probes, Life Technologies). Simultaneously, the dynamics of microarchitecture of unlabelled collagen fibers was monitored using reflective mode of CLSM. ${ }^{3,12}$ Scanning electron microscopy (SEM) of dehumidified 3D collagen coated with gold particles was conducted on a QUANTA FEG200 SEM system (FEI, Hillsboro, OR) under high vacuum conditions.

\section{Individual Cell Tracking and Data Analysis}

We monitored cell migration in a row of volume-ofviews spreading from the interface between the two gels to the outskirt of the gel, as illustrated in Fig. 1a. The volumes were taken $50 \mu \mathrm{m}$ above the dish bottom and $50 \mu \mathrm{m}$ below the top surface of the gel, each measuring $X \times Y \times Z=647 \times 647 \times 100 \mu \mathrm{m}^{3}$, with $5 \mu \mathrm{m}$ interval between adjacent focal planes. From these $Z$-stacks of confocal fluorescence images, 3D reconstruction and cell segmentation were carried out in Imaris (Bitplane, Zurich, Switzerland). Using the built-in particle-tracking algorithm in Imaris, we generated time-lapse cell tracks automatically with close human supervision. Tracks with $1500 \mathrm{~s}$ or longer duration were selected and corrected for drift whenever necessary. Cell invasion speed was averaged throughout the tracking duration and a total displacement vector for each cell was derived from the $3 \mathrm{D}$ cell position at each time point using MATLAB (The MathWorks, Inc., Natick, MA). Since the gel was constrained by the well, yielding no global gel deformation, tracking cells in absolute coordinate system faithfully reflects cell movement relative to the gel.

\section{RESULTS}

As we shall show, by monitoring 3D cancer cell migration into the originally cell-free collagen using time-lapse imaging, we found that the migration pattern of a cell varied with its relative location in a wave of radially spreading cells. In particular, we found higher cell migration speed and straighter tracks on the front of the cell spreading wave or "cell migration front". In addition, the matrix regions of increased cell population density were correlated with lower cell movement speed. We also assessed the collagen fiber network and found clustering of fibers in the vicinity of cells. Prior to cell invasion, the collagen fibers were self-assembled into uniformly distributed and randomly organized matrix. During cell migration, however, the cells reorganized the matrix fibers, as exemplified in the supporting Movies. Blocking actomyosin contractility impeded not only the migration of the cells, but also the cell-induced collagen matrix remodeling. These observations suggested a link between cell-induced modification of the micro-environment and the efficiency of contractile migration of cancer cells.

\section{Cell Speed and Directionality are Higher on the Invasion Front}

As expected for such highly metastatic cell line as MDA-MB-231, the cell population aggressively invaded the originally cell-free regions. After spending 4 5 days in the nested collagen gel supplied with complete culture media, cells started to escape from the original cell-seeding region and migrated into the outer acellular gel. More specifically, cells spread into a circular donut-shaped area surrounding the inner gel, reaching as far as $2.5-3.0 \mathrm{~mm}$ away from the two-gel interface after a 2-week period (Fig. 1a). Here, we analyze the cell migration behavior in the outer gel, as the study intends to simulate cell spreading involved in cancer metastasis. Generally, cells accomplished radial invasion through individual movement, without forming apparent cell-cell attachments. The average migration speed of the individual cells in 3D was 10$30 \mu \mathrm{m} / \mathrm{h}$, consistent with reported values for MDAMB-231 cells, ${ }^{39}$ albeit with a large population variance.

Interestingly, there are remarkable variations in MDA-MB-231 cell migration patterns, depending on the locations of the cells in the gel. Cells moved predominantly outwards on the cell migration front, leading to radial cell invasion into the previously cellfree regions. The leading cells were sparsely distributed and were more likely to move in the orientation perpendicular to the invasion front (Fig. 1b). Cells that trailed these leading cells, however, exhibit movements with significantly more random directions (Fig. 1c). We quantitatively characterized these distinct cell migration patterns by categorizing the cells according to the regions they were located. In particular, we refer to the regions on the front of cell invasion as "cell migration front" and to the vast region behind the front and closer to the boundary between the inner and 


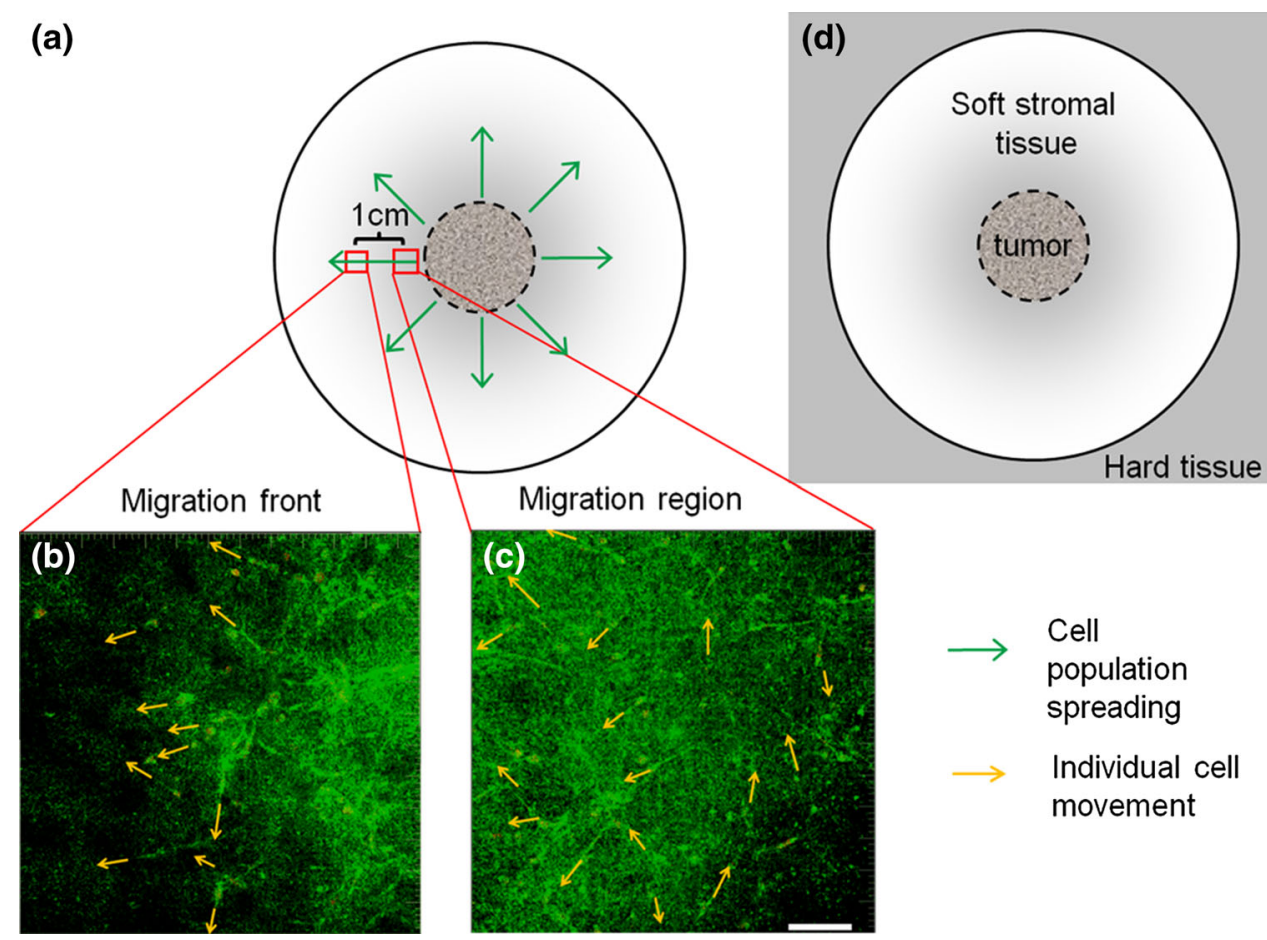

FIGURE 1. Cell migration in a 3D nested collagen gel model. Images were taken by CLSM and projected to 2D planes. (a) A schematic view of the nested gel model and the locations of volume-of-views (VoVs). Initially cells were seeded inside the region marked by the dashed circle. Cells then migrated from the inner gel in radial directions indicated by green arrows and formed a density gradient in the outer gel, as illustrated by the grey-level gradient. Distinct migration patterns are observed in the two VoV's marked by red squares. (b) and (c) Overlay of typical confocal reflective and fluorescence image of 3D collagen matrix (green) and the migrating MDA-MB-231 cells (red; yellow when overlaid with green pseudo-colored collagen), respectively, in the two VoVs. (b) Shows the "cell migration front", which encloses mostly the leading cells, and (c) shows "cell migration region", which is closer to the originally seeded inner gel. Black arrows point to the migration direction of individual cells within a time span of $5 \mathrm{~h}$. Scale bar $=50 \mu \mathrm{m}$. (d) A schematic illustration of how the nested gel model mimics the relative locations of tumor, soft stromal tissue, and hard tissue surrounding the soft tissue.

outer gel as "cell migration region". Figure 2 shows that the histogram of the migration speed of individual cell tracks (combined from multiple repeat assays) representing cell migration front is shifted to the right, compared to the histogram for the cell migration region, implying that cells were in general more motile in the former than in the latter. Indeed, when we further classified cells to be "motile" if they migrated faster than $15 \mu \mathrm{m} / \mathrm{h}$ (on average) in the outer gel, we found that only $53 \%$ of all cells in cell migration region were motile, whereas $72 \%$ of cells were motile in the cell migration front. It is important to note, however, that the cell-track migration speed was typically non-normally distributed with long tails. Nevertheless, the population-wide cell motility in the cell migration front was significantly larger than that in the cell migration region (Mann-Whitney $U$ test; $p<0.0001$ ), consistent with the disparity between the two medians shown in the legend of Fig. 2.

Cells moved predominantly horizontally throughout the $6 \mathrm{~h}$ time-lapse imaging, with negligible vertical (i.e., Z-direction) migration. We further quantified the long-term cell translocation directions based on time- averaged end-to-end displacement vectors of the cell tracks captured in each volume-of-view, as shown in Fig. 3. These Rose plots clearly reveal that cells on the cell migration front adopted predominantly directional movement, moving toward the negative $X$-direction, i.e., away from the original cell-seeding region. More specifically, between Day 5 and Day 13 after the start of the experiment, cells in the cell migration front continued to effectively invade the tissue surrounding the simulated tumor. In contrast, on Day 13, follower cells in the cell migration region did not show a significant net translocation as a population, as indicated by the diverse movement directionality and the negligible net population velocity.

\section{Collagen Fibers were Aligned with the Orientation of Cell Movement}

To better understand the underlying reason behind the significant population variance and distribution of cell migration speed, we investigated the local environment surrounding individual migrating cells. A closer inspection on the local behavior of each individual cell 


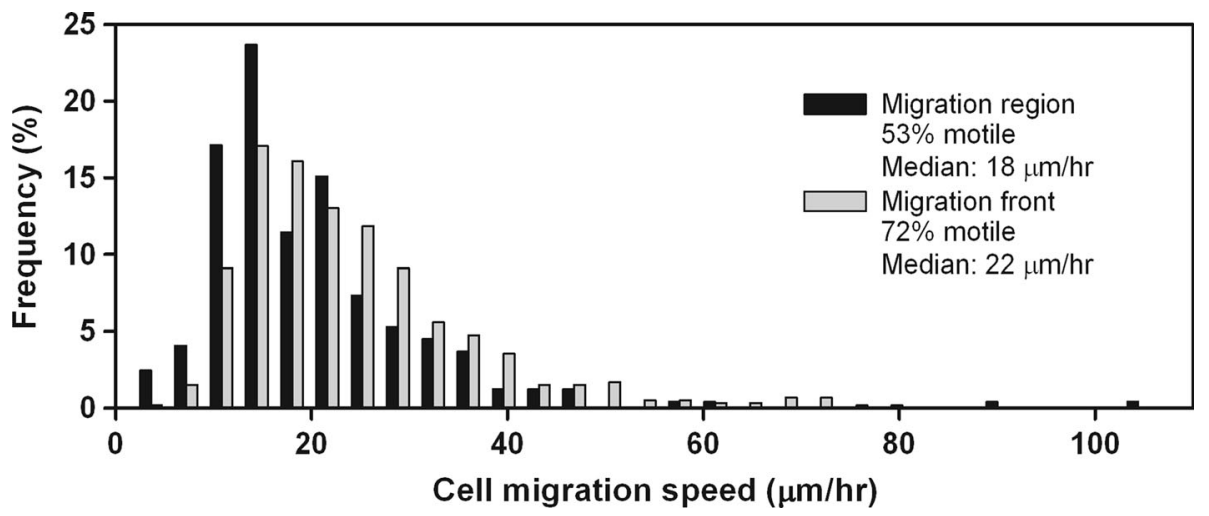

FIGURE 2. Cell speed on the 12th day of culture. We plot the histogram of the count frequency distribution of cell tracks at each level of cell migration speed in $1.5 \mathrm{mg} / \mathrm{mL}$ collagen gel. The statistics was based on 200-300 individual cell tracks. In the legend, "'motile" refers to the \% proportion of cell population that moved faster than $15 \mu \mathrm{m} / \mathrm{h}$ over its movement track. "Median" refers to the median cell speed in each category.

uncovered a consistent co-localization of contractile cell movement and collagen fiber realignment along the main axis of spindle-shaped cells. In line with previous observations for MDA-MB-231 cell line, ${ }^{6,18,23}$ Movies S1-S3 show typical examples that in the 3D collagen matrices the cells migrated in mesenchymal fashion, which typically relies on cell contractions. Interestingly, these cell contractions were accompanied by dynamic reorganization of collagen fibers around the migrating cells. Figures $4 \mathrm{a}-4 \mathrm{~d}$ show two snapshots of a migrating cell, with arrow-heads pointing to both the assembly of fibers around the pseudopods that preceded cell translocation and the alignment of fibers along the direction of cell migration. The local fiber movement relative to the whole matrix seemed to indicate non-uniform network straining, consistent with hypothesis of non-affine deformation in biopolymer networks. ${ }^{4,5}$ SEM imaging further confirmed that, at the leading and trailing ends of a cell, fibers were taut and aligned with the elongated cell body (arrow, Fig. 4e). In addition to fluorescence imaging of cells, simultaneous reflective confocal imaging of the collagen fiber network revealed that the local deformation of collagen matrix frequently accompanied cell contraction and, in some cases, the retraction of cell trailing edge from the matrix. In contrast to the nonaffine fiber matrix deformation in the proximity of cells, collagen fibers in the cell-free region appeared to move uniformly as a whole network, as for instance shown on the top-left corner region in Movie S3.

\section{ROCK-Mediated Cell Contractility is Required for} Matrix Reorganization and Effective 3D Cell Migration

We further investigated the role of cell contraction, in the form of active actomyosin machinery, in the extent of matrix reorganization and the resulting cell migration. In particular, we focused on Rho kinases
(ROCKs), which are serine/threonine kinases that mediate myosin-controlled cell contraction and migration ${ }^{14,40}$ and which have been previously suggested to play an important role in cell-induced collagen matrix realignment and global matrix contraction. $^{24}$ We introduced ROCK inhibitor Y27632 to the media on Day 6 of the experiment, after MDA-MB-231 cells had adapted themselves to the 3D collagen environment. We found that the inhibition of ROCK activity induced dendritic cell morphology (Figs. 5a, 5b), suggesting a dramatic, although not unexpected, change in the mechanism of cell contractility. More importantly, the extent of matrix reorganization around cells was reduced, as evidenced by the random orientation and uniform distribution of matrix fibers shown in Fig. 5a, which resembled that of intact collagen meshwork. Furthermore, histograms of cell migration speed suggest that this reduction in the extent of matrix reorganization was accompanied by significantly reduced cell speed in 3D collagen gel (Fig. 5c). Again, we categorized cells according to their motility, defining cells that migrated faster than $15 \mu \mathrm{m} /$ $\mathrm{h}$ as "motile cells", and found that only $7 \%$ of the cells are in the "motile" group after drug treatment, compared to $38 \%$ in the control experiment. The median cell speed also decreased from 14 to $7 \mu \mathrm{m} / \mathrm{h}$. These results suggest a direct link between ROCK-mediated cell contractility, cell-induced matrix reorganization, and the effectiveness and rate of cell migration.

\section{Modification of the Collagen Matrix Limits Cell Migration}

The cell-induced modification of collagen fiber network was indeed found to directly alter cell migration behaviors. An example is shown in Movie S3-a, in which it can be seen that a cell in a cell-sparse matrix 


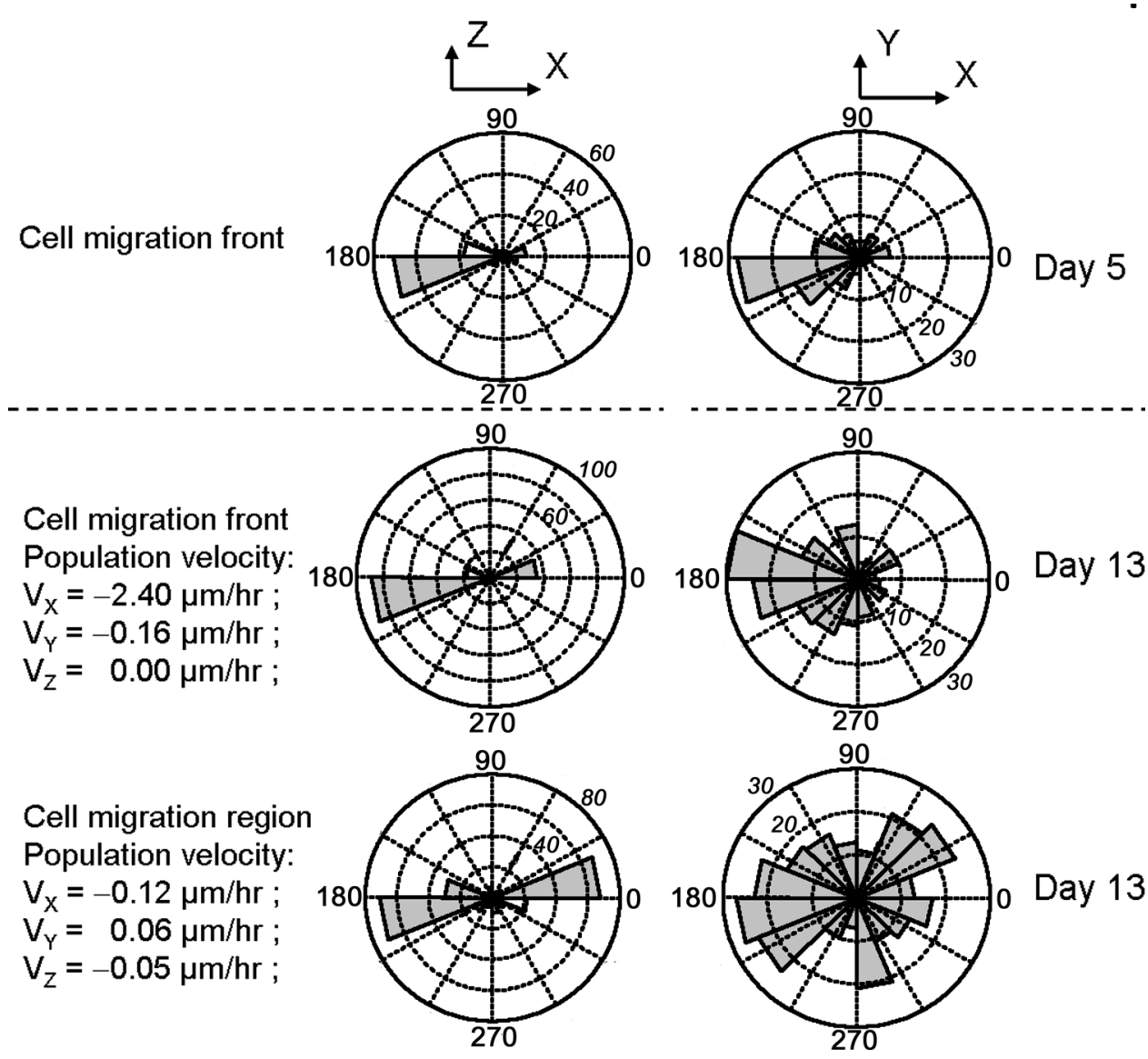

FIGURE 3. Rose plots of cell movement directionality. The directions of individual cell movements were plotted as a function of angles (in degrees) in the $X-Z$ plane (left panel) and $X-Y$ plane (right panel). The track counts are shown in italics. In all cases shown in this figure, the original cell-seeding region is to the right of the observed VoV. "Cell migration region" refers to the VoV nearest to the interface between inner and outer gel (region "C" in Fig. 1); "Cell migration front" refers to the VoV that included the foremost moving cells, but excluding few outliers (region "B" in Fig. 1). On Day 5, cells had not moved far out of the original cell seeding region (most cells were found in region " $C$ " in Fig. 1), so we did not differentiate between "Cell migration region" and "Cell migration front" regions. By Day 13, however, the plots show a clear contrast between the two regions. The cell population velocity shown for Day 13 were averages of all cell-tracks in a VoV (100-200 cell tracks) located on "Cell migration front" and "Cell migration region" within a time-span of $6 \mathrm{~h}$. Cartesian (rather than radial) coordinate system was used to emphasize that, while the cell population migration is predominantly radial with respect to the center of the cell-seeding area, the directionality of individual cell migration varies depending on the relative location of the cell within the population.

region (indicated by arrows) moved much more easily than a cell in a region populated with many cells (Movie S3-b, indicated by arrow-head). The latter cell appeared to be actively moving but was entrapped by the collagen network that had been surrounded by neighbor cells. Movie S2 shows another example where cells adhered to, rearranged and deformed collagen fibers during active movement. After a failed attempt to pull the fibers between the two cells, the lower cell retracted quickly and receded instead of moving towards the upper cell. As the lower cell withdrew, some fibers between these two cells dissociated from the local cluster of fibers.

Based on the findings described in the above sections, we speculated that the presence of other cells in the population might affect cell motility through mechanical connectivity of the matrix network. A simple test to check this hypothesis can be performed by quantifying the migration behavior of cells seeded with different seeding densities. We carried this out for cell seeding densities of 25,50 , and 100 thousand cells/ $\mathrm{mL}$ and found that, consistent with the above hypothesis, cells moved significantly faster in the gel regions of lower cell population densities than in the more crowded matrix (Fig. 6). The current study focused on the migration behavior of malignant cancer cells, with the highly invasive MDA-MB-231 cells as a model system. As a control, we have also performed tests with benign breast tumor MCF-10A cells under the same experimental conditions. As expected, the migration speed of these cells was significantly lower than that of MDA-MB-231, and we did not find any 

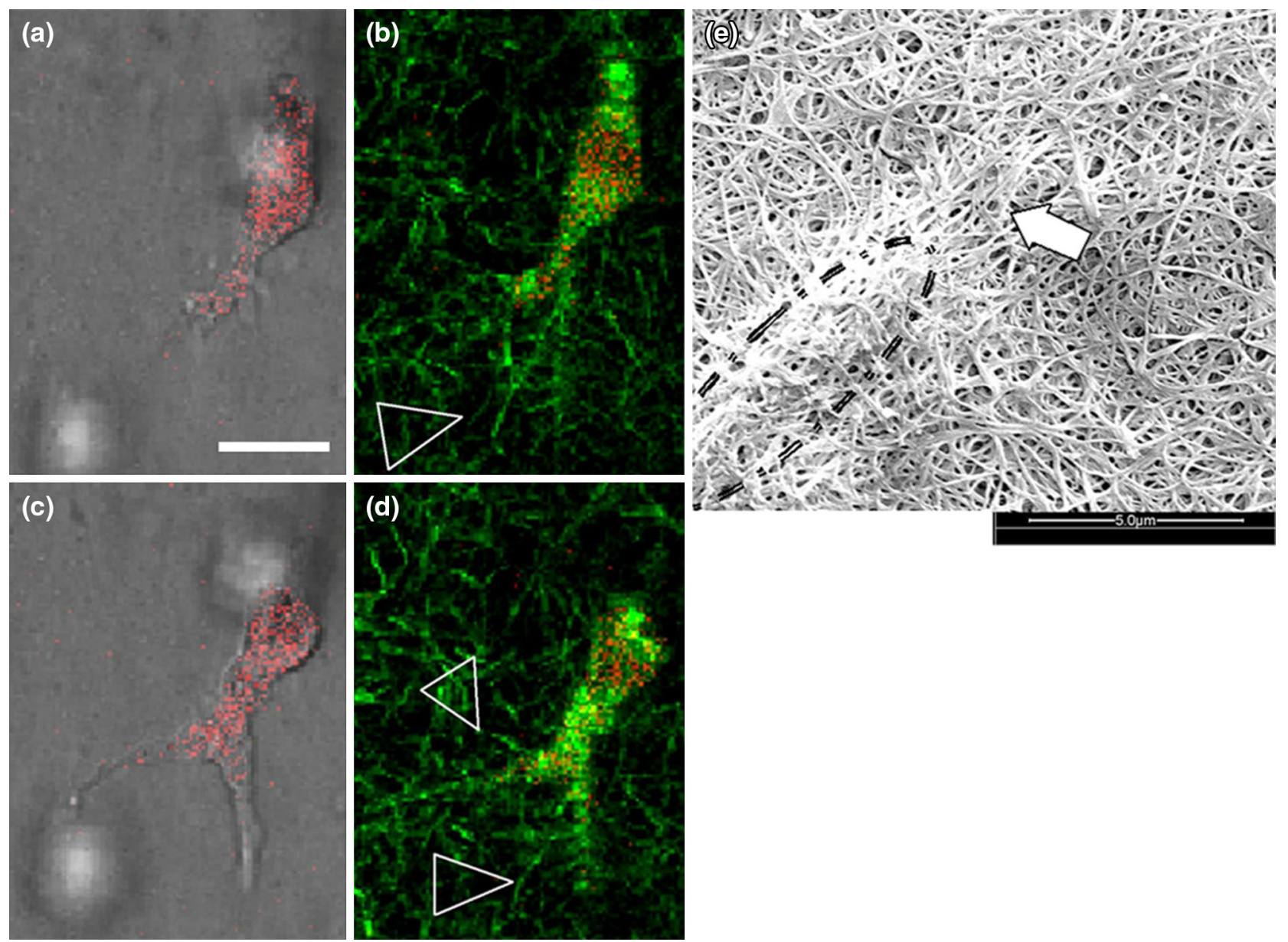

FIGURE 4. Movement of individual cell and the fibers reorganization in the collagen matrix. (a) and (b) Time = 0 ; (c) and (d) Time = $10 \mathrm{~min}$. (a) and (c): overlay of Differential Interference Contrast (DIC) and confocal fluorescence images; (b) and (d): overlay of confocal fluorescence signal of cells (red) and back-scattering signal of collagen fibers (green). Around cell bodies, fibers were frequently restructured to form bundles near cell protrusions at the leading-edge, or were aligned along the pseudopods (arrowhead). Scale bar $=20 \mu \mathrm{m}$. (e): SEM image of a MDA-MB-231 cell embedded in collagen matrix, under dehydrated, vacuum condition. The cell body is outlined with a double-dashed line. The fibers closely wrapping the cell are taut and aligned either parallel or transverse to the main axis of the cell body, indicating that the fibers are under tension. The low curvature of fibers in (b), (d) and (e) suggests fibers are stiff in the network.

evidence for spatially distinct migration patterns (data not shown). Although this result may suggest that the spatially distinct cell migration behavior is a property of invasive cancer cells, further studies with different cell lines will be needed to confirm such generalization.

\section{DISCUSSION}

In this study, we built a 3D nested gel migration assay and quantitatively assessed the migration behavior of highly invasive breast cancer cells. Compared to the floating collagen gel model, ${ }^{17}$ our adherent $3 \mathrm{D}$ collagen model is more physiologically relevant and better resembles the physical characteristics of cancer invasion. Geometrically, the inner gel with a high cancer cell density mimics the original tumor, while the outer gel mimics the stromal connective tissue invaded by the cancer cells. This outer gel is mechanically supported by the outer rim of the well, just as soft connective tissues are supported by the relatively more rigid tissues (Fig. 1d). Our quantitative analysis shows that this nested gel configuration results in a predominantly horizontal cancer cell population spreading in a radial-spreading manner with negligible vertical displacement. This can be explained by the fact that the top surface of the outer gel is not anchored to any solid structure and thus cannot sustain vertical contractions by the cells, whereas the sides are mechanically supported by the walls. In contrast, earlier studies using fully-enclosed 3D collagen gel found that the vertical migration of fibroblasts was comparable with the horizontal migration in magnitude. ${ }^{9}$ These findings suggest the crucial role of cell contractility in $3 \mathrm{D}$ cell migration. 

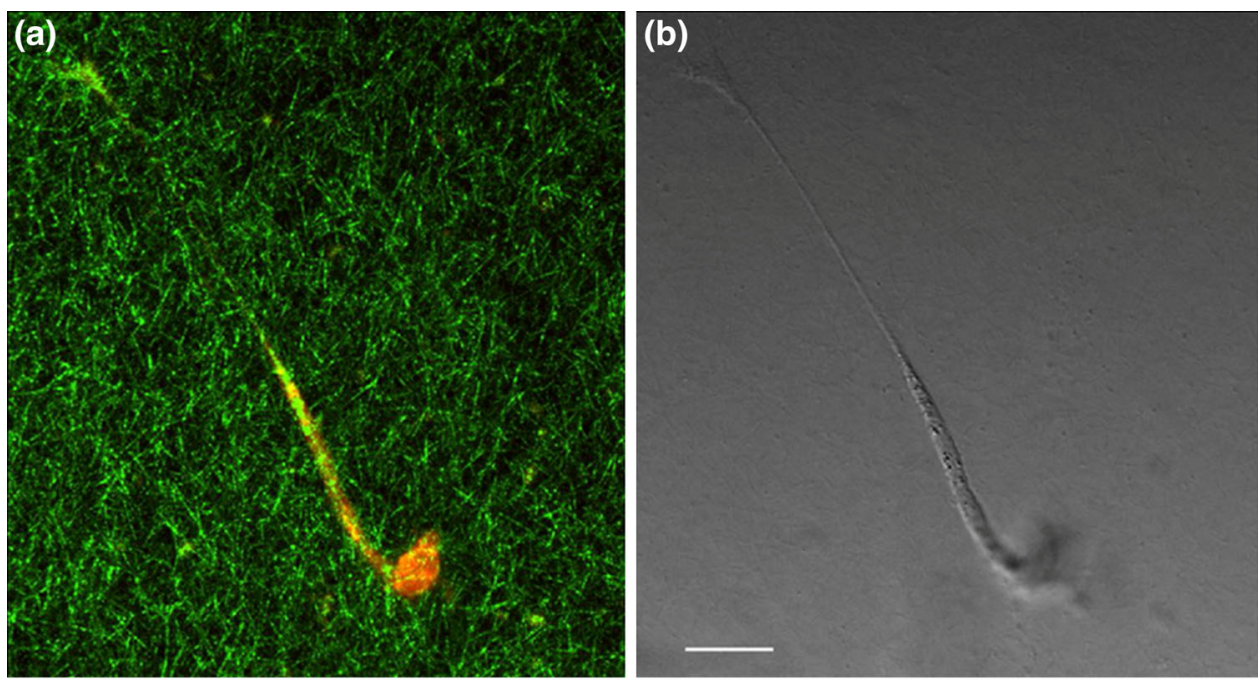

(c)

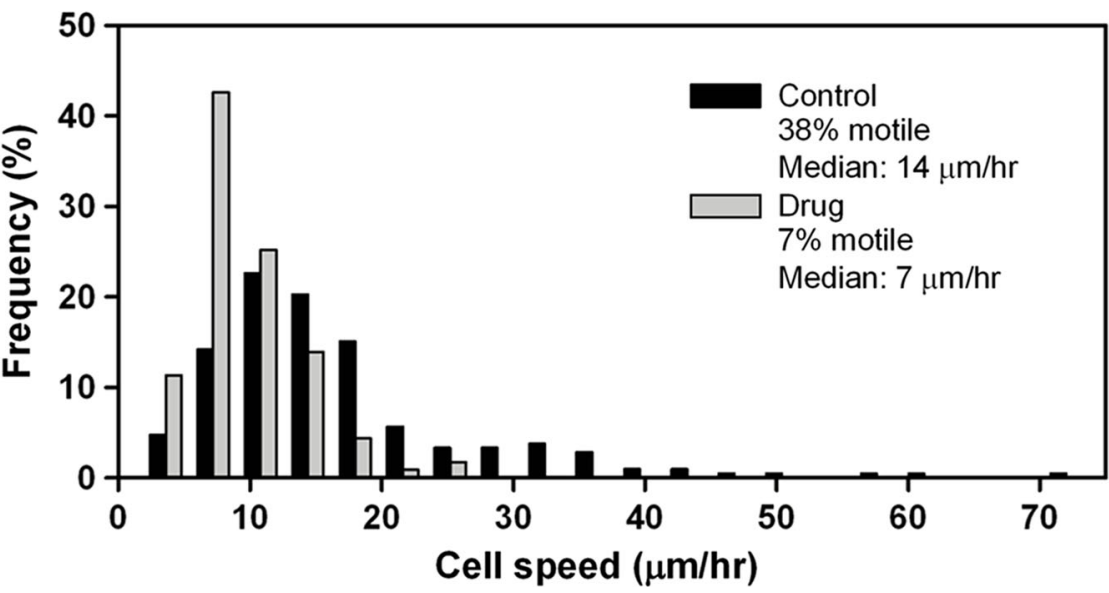

FIGURE 5. Typical morphology and cell migration behavior of MDA-MB-231 cell in 3D collagen gel under ROCK inhibitor treatment. (a): Overlay of collagen (green) and CMTMR stained cells (red) with maximum intensity projection from a 3D-stack of confocal reflectance and fluorescence images, respectively. (b): DIC image of the cell. Scale bar $=20 \mu \mathrm{m}$. (c): Histogram of average 3D speed of cells migrating into $1.5 \mathrm{mg} / \mathrm{mL}$ collagen gel. "Drug" refers to the cell migration under ROCK inhibitor treatment; "Control" refers to the control experiment. "Motile cells" refer to the proportion (\%) of cells that moved faster than $15 \mu \mathrm{m} / \mathrm{hr}$. "Median" denotes the median of cell speed.

Indeed, inhibition of ROCK-mediated cell contractility significantly decreases the ability of the cells to migrate. Furthermore, this cell contractility must be mechanically supported by the surrounding matrix. Consequently, insufficient mechanical support leads to abolition of migration, as indicated by the negligible vertical cell displacement in our system.

Within the radially-spreading cancer cell population, we observed distinct cell migration patterns: a fast, directionally persistent cells on the migration front and a slow, less directed cells behind those leading cells. It is important to note, however, that this difference is not linked to specific locations in the gels. Rather, the difference stems from the relative positions of the cells in the migration of the cell population. As Fig. 3 suggests, the pioneer ("leader") cells moved in a more directionally persistent manner on Day 5 (top panel) than the "follower" cells did on Day 13 (bottom panel), even though the data were obtained from the same location in the gel. The difference is that, by Day 13, "leader cells" had moved past this region, leaving a heavily modified matrix region to be explored and migrated into by the "follower cells". These distinct migratory patterns are also independent of the stiffness of the gels; they were observed in all our experiments regardless of collagen concentration and pore size. The previous suggestion that matrix mechanics plays a role in cell migration strategy is therefore incomplete if one attempts to fully understand cancer cell migration at both population level and individual cell level.

There are several factors that could contribute to the radial spreading of the cancer population and the position-dependent migration patterns. Factors that are in spatiotemporal equilibrium, such as chemotaxis, 


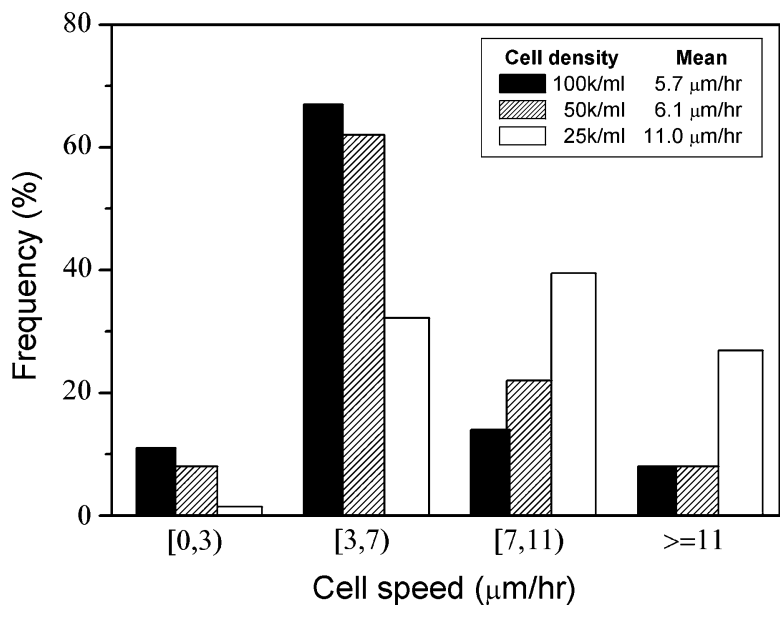

FIGURE 6. Cell migration speed depended on cell population density. Cells were seeded uniformly in 3D collagen gel with different cell seeding density $(25,50$ and $100 \mathrm{k}$ cells $/ \mathrm{mL})$ and were allowed to migrate in random manner. Lower cell seeding density resulted in higher cell motility. The collagen gel protein concentration was $2.5 \mathrm{mg} / \mathrm{mL}$.

can however be ruled out. First, a long term gradient of oxygen or nutrients over time scales of hours or even days is difficult to sustain in collagen gel systems with pore size on the order of microns like in our system. For example, a previous study reported that it took mere minutes for thrombin to diffuse through a collagen gel of $1.5 \mathrm{~cm}$ in diameter and $0.4 \mathrm{~cm}$ thick, ${ }^{26}$ which are comparable to the size of the collagen gels used in our study. Second, although these factors may in principle cause general spreading of cell population, such factors cannot account for the spatially distinct migration patterns observed in our study. On the other hand, there are factors related to biochemical signaling, such as haptotaxis (caused by non-uniform distribution of matrix-immobilized cell-adhesion ligands) and asymmetry in the cell-cell cross-talk and signaling, that may explain the difference between the migration patterns between the cells on the cell migration front and the cells on the cell migration region. Alternatively, polarized cell surface receptor distribution and cell paracrine signals ${ }^{42}$ may also play a role in the migration mechanisms of the cells. Although these factors can create local asymmetry of signaling, which in principle can account for the spatially distinct migration behavior, they cannot explain the overall spreading trend of the cell population. We hypothesize that mechanics-based factor can in fact be the most universal explanation to all our observations. In particular, we have noticed that occasionally, when some part of the periphery of the outer gel detached from the dish wall, cells ceased migration into that region, akin to the case of floating gel, ${ }^{33}$ while they continued radial migration into other regions. A recent numerical modeling of cell migration in 3D gel that explicitly took into account cell contractility mechanisms also shows that cells in a uniform gel tend to move towards a constrained boundary. ${ }^{2}$ Another numerical simulation provided quantitative understanding of the physical process that cells remodel the surrounding matrix during invasion. ${ }^{20}$ These observations lend support to mechanics-based explanation to our findings.

While a thorough space- and time-resolved biochemical and biophysical studies are clearly needed to establish the specific molecular mechanism behind these speculated factors, the observation of contractility-mediated physical remodeling of the matrix offers an alternative perspective that reveal the important role of local matrix microenvironment. The modification to the ECM can be achieved through collagen fiber reorganization. In our $3 \mathrm{D}$ collagen model, we observed that cells extended out dynamic pseudopods into multiple pericellular regions of the matrix, reorganized the collagen network locally, and finally followed one of these pseudopods to migrate in a particular direction. This finding correlates well with the previous observation that dynamic local remodeling of collagen hydrogel by migrating tumor cells was most pronounced around the cell leading and trailing edges. ${ }^{1,25}$ Importantly, since collagen fibers were clustered around each cell, the resulting bundling of collagen fibers further caused the originally homogeneous ECM to be effectively partitioned into smaller sub-regions by cell bodies, as illustrated in Figs. 7a, 7b. Moreover, the segmentation of the fiber network became more significant with higher cell density in ECM. In contrast, collagen fibers remained randomly distributed and interwoven network ahead of the outward-moving cells on the cell migration front (Fig. 1b).

We note that in this study we used pepsin-treated collagen-I. It has been shown that $3 \mathrm{D}$ gels formed from pepsin-treated collagen are not covalently cross-linked, thus allowing for MMP-independent cell invasion. ${ }^{35}$ The cell-induced matrix modification observed in our study is likely to be due to the interplay of multiple mechanisms, including matrix degradability, which is related to the degree of cross-linking in the matrix. In the future, it would be interesting to directly test the influence of different levels of matrix degradability, for instance using pepsinized and non-pepsinized collagen with similar network structure, and matrix composition, for instance using Matrigel, on the spatiotemporal variability and regulation of cell invasion. It is important to consider, however, that the self-assembly kinetics of collagen gel is strongly dependent on the presence of the telopeptides (among other things), thus making the final network structure highly variable. ${ }^{50}$ Since cell migration behavior is strongly affected by network structure, to be able to make a direct 

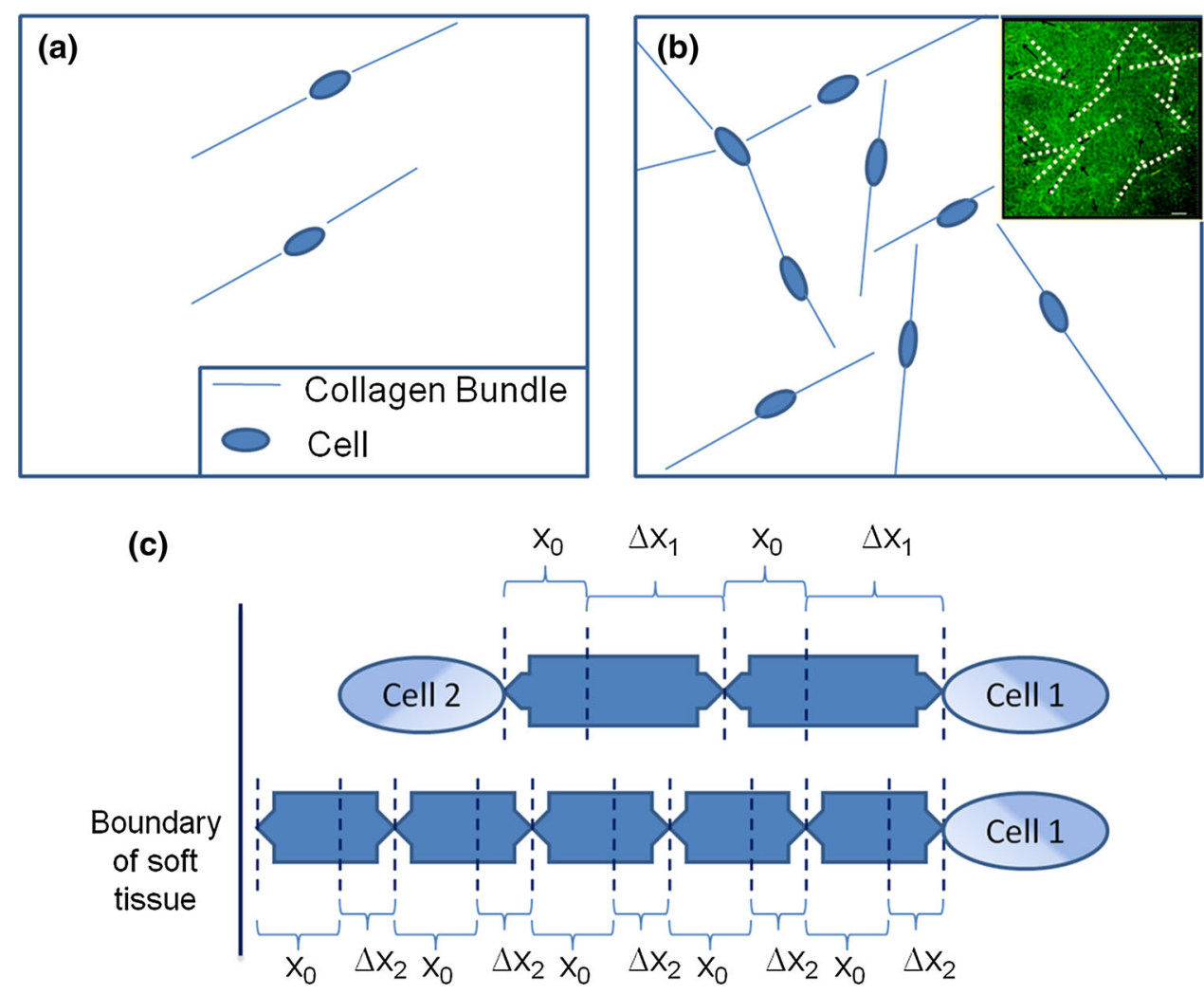

FIGURE 7. Schematics of the cell-induced segmentation of collagen matrix. (a) Negligible segmentation, when cells are sparsely distributed. (b): Intensive segmentation, where the matrix area has been effectively divided into smaller regions. The inset shows an example, based on the image in Fig. 1c, where the segmentation of matrix by cells are highlighted with dotted lines. (c): Simplified 1D spring model to illustrate the mechanics of cell contraction. We made an assumption that the ECM can be well described as an elastic continuum. A bundle of collagen fibers as a force-bearing unit in the matrix is simplified to a spring segment, and the whole collagen matrix is modeled as springs in serial connections. Each spring segment has equal original length, $x_{0}$, and equal spring constant. The elongation due to cell contraction is $\Delta x_{1}$ for fibers in the matrix compartmented by cells and $\Delta x_{2}$ for the cell-free matrix, respectively. The same magnitude of cell-exerted force resulted in a larger extension in each spring segment in a cell-segmented matrix than in a matrix region without the presence of cells, i.e., $\Delta x_{1} / x_{0}>\Delta x_{2} / x_{0}$. In cases when $\Delta x_{1} / x_{0}$ exceeded the elastic range, cell contraction of the matrix may not be successful.

comparison, one would ideally need to find a set of independent experimental conditions $(\mathrm{pH}$, ionic strength, etc.) such that the final network structures of the different collagens are closely comparable.

Previous in vivo and in vitro studies found evidence that, within an invading cell colony, a leader cell can cleave and remodel fibers, thereby generating tube-like microtracks that serve as paths of least resistance for the following cells. ${ }^{13,44}$ In this case, the leader cells, which can be activated stromal fibroblasts or cancer cells themselves, ${ }^{15}$ initiate and promote collective invasion of follower cells in a cell-cell contact dependent manner. This strand-like collective invasion widens the microtracks to become macropatterns that further enables directed invasion of the following cell mass. ${ }^{49}$ In contrast, by using the highly invasive MD-MBA-231 cells, our work focused on the invasion behavior of single cells in a population. These cells largely favor mesenchymal, cell contact-independent migration, even at relatively large cell density. Our finding that the cells in the migration front invade in a much more persistent and directed manner compared to the "follower cells" may seem somewhat surprising, and therefore highlights the ability of cells to adapt their migratory strategy to their surrounding physical environment.

Taking these discussions together, our earlier findings that link cell migration to matrix reorganization can be understood in terms of a mechanical model of contractile cell migration in soft collagen gel on the micro-scale. It is known that the mesenchymal migration of cancer cells is contractile and is closely associated with collagen gel straining and relaxation, as reported, e.g., by Bloom et al. ${ }^{1}$ Cells are also sensitive to local mechanical perturbation that can be transmitted to cells through the $\mathrm{ECM}^{29}{ }^{29}$ because of the cells' local mechano-sensing ability. It is therefore likely that when the elastic contractility of the local matrix around a cell is interfered, the cell's ability to locomote may be adversely affected. In the presence of other cells, the pronounced segmentation of the ECM into sub-regions in fact reduces the

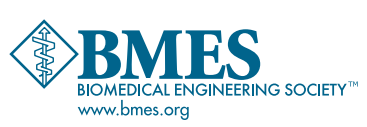


area of ECM around a cell that can be freely deformed. As a result, the same level of cell contraction force was less likely to induce non-elastic local deformation of the matrix, as illustrated in Fig. 7c. For example, in a cell cluster, a cell could be trapped in the dense fibers near other cells, such as what the cell on the far-right corner of the screen of Movie 1 experienced towards the end of the movie. As one consequence of the above mechanical picture, the reduced directional cell migration in the cellmodified matrix areas could arise from the segmentation of collagen matrix. Meanwhile, there are fewer cells in the matrix ahead of the cell spreading front; therefore, the path towards cell-free region presents the path of least resistance for a cell. This might be responsible for the spreading of invasive tumor cells in ECM.

In summary, our study emphasizes the need for a micromechanical understanding to analyze spontaneous cancer cell migration and physical remodeling of the local collagen matrix. We focused on how modification of the matrix fed back to influence cell migration. These modifications result in distinct cell migration patterns on the migration front and its wake. It would therefore be highly useful to develop techniques that can reliably allow one to monitor, in space and time, the local mechanical properties of the matrix surrounding individual cells. ${ }^{51}$ Moreover, despite the intriguing invasion behavior of cancer cells themselves, the role of fibroblasts in priming local ECM microenvironment for cancer invasion has been increasingly recognized. ${ }^{44}$ It will be highly instructive to conduct co-culture of fibroblasts and invasive cells and quantitative assays such as those performed in this study to further understand the potential feedback mechanism between the two cell types.

\section{ELECTRONIC SUPPLEMENTARY MATERIAL}

The online version of this article (doi:10.1007/s12195014-0324-0) contains supplementary material, which is available to authorized users.

\section{ACKNOWLEDGMENTS}

Supports provided by the Global Enterprise for Micro-Mechanics and Molecular Medicine (GEM4) and the NUS Graduate School for Integrative Sciences and Engineering are gratefully acknowledged.

\section{CONFLICT OF INTEREST}

W. Sun, N. A. Kurniawan, A. P. Kumar, R. Rajagopalan, and C. T. Lim declare that they have no conflicts of interest.

\section{ETHICAL STANDARDS}

No human or animal studies were carried out by the authors for this article.

\section{REFERENCES}

${ }^{1}$ Bloom, R. J., J. P. George, A. Celedon, S. X. Sun, and D. Wirtz. Mapping local matrix remodeling induced by a migrating tumor cell using three-dimensional multipleparticle tracking. Biophys. J. 95:4077-4088, 2008.

${ }^{2}$ Borau, C., R. D. Kamm, and J. M. Garcia-Aznar. Mechano-sensing and cell migration: a 3D model approach. Phys. Biol. 8:066008, 2011.

${ }^{3}$ Brightman, A. O., B. P. Rajwa, J. E. Sturgis, M. E. McCallister, J. P. Robinson, and S. L. Voytik-Harbin. Time-lapse confocal reflection microscopy of collagen fibrillogenesis and extracellular matrix assembly in vitro. Biopolymers 54:222-234, 2000.

${ }^{4}$ Broedersz, C. P., K. E. Kasza, L. M. Jawerth, S. Munster, D. A. Weitz, and F. C. MacKintosh. Measurement of nonlinear rheology of cross-linked biopolymer gels. Soft Matter 6:4120-4127, 2010.

${ }^{5}$ Broedersz, C. P., M. Sheinman, and F. C. MacKintosh. Filament-length-controlled elasticity in 3D fiber networks. Phys. Rev. Lett. 108:078102, 2012.

${ }^{6}$ Castello-Cros, R., D. R. Khan, J. Simons, M. Valianou, and E. Cukierman. Staged stromal extracellular 3D matrices differentially regulate breast cancer cell responses through PI3K and betal-integrins. BMC Cancer 9:94, 2009

${ }^{7}$ Condeelis, J., and J. E. Segall. Intravital imaging of cell movement in tumours. Nat. Rev. Cancer 3:921-930, 2003.

${ }^{8}$ Decaestecker, C., O. Debeir, P. Van Ham, and R. Kiss. Can anti-migratory drugs be screened in vitro? A review of 2D and 3D assays for the quantitative analysis of cell migration. Med. Res. Rev. 27:149-176, 2007.

${ }^{9}$ Dickinson, R. B., S. Guido, and R. T. Tranquillo. Biased cell migration of fibroblasts exhibiting contact guidance in oriented collagen gels. Ann. Biomed. Eng. 22:342-356, 1994.

${ }^{10}$ Entschladen, F., T. L. Drell, K. Lang, K. Masur, D. Palm, P. Bastian, B. Niggemann, and K. S. Zaenker. Analysis methods of human cell migration. Exp. Cell Res. 307:418426, 2005.

${ }^{11}$ Even-Ram, S., and K. M. Yamada. Cell migration in 3D matrix. Curr. Opin. Cell Biol. 17:524-532, 2005.

${ }^{12}$ Friedl, P. Dynamic imaging of cellular interactions with extracellular matrix. Histochem. Cell Biol. 122:183-190, 2004.

${ }^{13}$ Friedl, P., and K. Wolf. Tube travel: the role of proteases in individual and collective cancer cell invasion. Cancer Res. 68:7247-7249, 2008.

${ }^{14}$ Fukata, Y., M. Amano, and K. Kaibuchi. Rho-Rho-kinase pathway in smooth muscle contraction and cytoskeletal reorganization of non-muscle cells. Trends Pharmacol. Sci. 22:32-39, 2001

${ }^{15}$ Gaggioli, C., S. Hooper, C. Hidalgo-Carcedo, R. Grosse, J. F. Marshall, K. Harrington, and E. Sahai. Fibroblast-led collective invasion of carcinoma cells with differing roles for RhoGTPases in leading and following cells. Nat. Cell Biol. 9:1392-1400, 2007. 
${ }^{16}$ Goetz, J. G., S. Minguet, I. Navarro-Lérida, J. J. Lazcano, R. Samaniego, E. Calvo, M. Tello, T. Osteso-Ibáñez, T. Pellinen, A. Echarri, A. Cerezo, A. J. P. Klein-Szanto, R. Garcia, P. J. Keely, P. Sánchez-Mateos, E. Cukierman, and M. A. Del Pozo. Biomechanical remodeling of the microenvironment by stromal Caveolin-1 favors tumor invasion and metastasis. Cell 146:148-163, 2011.

${ }^{17}$ Grinnell, F., L. B. Rocha, C. Iucu, S. Rhee, and H. Jiang. Nested collagen matrices: a new model to study migration of human fibroblast populations in three dimensions. Exp. Cell Res. 312:86-94, 2006.

${ }^{18}$ Han, J., H. Chang, O. Giricz, G. Y. Lee, F. L. Baehner, J. W. Gray, M. J. Bissell, P. A. Kenny, and B. Parvin. Molecular predictors of 3D morphogenesis by breast cancer cell lines in 3D culture. PLoS Comput. Biol. 6:e1000684, 2010.

${ }^{19}$ Hanahan, D., and R. A. Weinberg. The hallmarks of cancer. Cell 100:57-70, 2000.

${ }^{20}$ Harjanto, D., and M. H. Zaman. Modeling extracellular matrix reorganization in $3 \mathrm{D}$ environments. PLoS ONE 8:e52509, 2013.

${ }^{21}$ Jones, P. A., and Y. A. De Clerck. Extracellular matrix destruction by invasive tumor cells. Cancer Metastasis Rev. 1:289-317, 1982.

${ }^{22}$ Junkin, M., and P. K. Wong. Probing cell migration in confined environments by plasma lithography. Biomaterials 32:1848-1855, 2011.

${ }^{23}$ Kenny, P. A., G. Y. Lee, C. A. Myers, R. M. Neve, J. R. Semeiks, P. T. Spellman, K. Lorenz, E. H. Lee, M. H. Barcellos-Hoff, O. W. Petersen, J. W. Gray, and M. J. Bissell. The morphologies of breast cancer cell lines in three-dimensional assays correlate with their profiles of gene expression. Mol. Oncol. 1:84-96, 2007.

${ }^{24}$ Kim, A., N. Lakshman, and W. M. Petroll. Quantitative assessment of local collagen matrix remodeling in 3-D culture: the role of Rho kinase. Exp. Cell Res. 312:3683-3692, 2006.

${ }^{25}$ Koch, T. M., S. Münster, N. Bonakdar, J. P. Butler, and B. Fabry. 3D traction forces in cancer cell invasion. PLoS ONE 7:e33476, 2012.

${ }^{26}$ Kolodney, M. S., and E. L. Elson. Correlation of myosin light chain phosphorylation with isometric contraction of fibroblasts. J. Biol. Chem. 268:23850-23855, 1993.

${ }^{27}$ Kurniawan, N. A., S. Enemark, and R. Rajagopalan. The role of structure in the nonlinear mechanics of cross-linked semiflexible polymer networks. J. Chem. Phys. 136:065101, 2012.

${ }^{28}$ Kurniawan, N. A., L. H. Wong, and R. Rajagopalan. Early stiffening and softening of collagen: interplay of deformation mechanisms in biopolymer networks. Biomacromolecules 13:691-698, 2012.

${ }^{29}$ Lo, C. M., H. B. Wang, M. Dembo, and Y. L. Wang. Cell movement is guided by the rigidity of the substrate. Biophys. J. 79:144-152, 2000.

${ }^{30}$ Menezes, G. C., M. Miron-Mendoza, C. H. Ho, H. Jiang, and F. Grinnell. Oncogenic Ras-transformed human fibroblasts exhibit differential changes in contraction and migration in 3D collagen matrices. Exp. Cell Res. 314:3081-3091, 2008.

${ }^{31}$ Menon, S., and K. A. Beningo. Cancer cell invasion is enhanced by applied mechanical stimulation. PLOS ONE 6:e17277, 2011.

${ }^{32}$ Miller, E. D., K. Li, T. Kanade, L. E. Weiss, L. M. Walker, and P. G. Campbell. Spatially directed guidance of stem cell population migration by immobilized patterns of growth factors. Biomaterials 32:2775-2785, 2011.
${ }^{33}$ Miron-Mendoza, M., J. Seemann, and F. Grinnell. Collagen fibril flow and tissue translocation coupled to fibroblast migration in 3D collagen matrices. Mol. Biol. Cell 19:20512058, 2008.

${ }^{34}$ Nelson, C. M., and M. J. Bissell. Modeling dynamic reciprocity: engineering three-dimensional culture models of breast architecture, function, and neoplastic transformation. Semin. Cancer Biol. 15:342-352, 2005.

${ }^{35}$ Packard, B. Z., V. V. Artym, A. Komoriya, and K. M. Yamada. Direct visualization of protease activity on cells migrating in three-dimensions. Matrix Biol. 28:3-10, 2009.

${ }^{36}$ Petroll, W. M., and L. Ma. Direct, dynamic assessment of cell-matrix interactions inside fibrillar collagen lattices. Cell Motil. Cytoskeleton 55:254-264, 2003.

${ }^{37}$ Petroll, W. M., L. Ma, and J. V. Jester. Direct correlation of collagen matrix deformation with focal adhesion dynamics in living corneal fibroblasts. J. Cell Sci. 116:1481-1491, 2003.

${ }^{38}$ Provenzano, P. P., D. R. Inman, K. W. Eliceiri, J. G. Knittel, L. Yan, C. T. Rueden, J. G. White, and P. J. Keely. Collagen density promotes mammary tumor initiation and progression. BMC Med. 6:11, 2008.

${ }^{39}$ Provenzano, P. P., D. R. Inman, K. W. Eliceiri, S. M. Trier, and P. J. Keely. Contact guidance mediated threedimensional cell migration is regulated by Rho/ROCKdependent matrix reorganization. Biophys. J. 95:53745384, 2008.

${ }^{40}$ Riento, K., and A. J. Ridley. Rocks: multifunctional kinases in cell behaviour. Nat. Rev. Mol. Cell Biol. 4:446-456, 2003.

${ }^{41}$ Sahai, E., and C. J. Marshall. Differing modes of tumour cell invasion have distinct requirements for Rho/ROCK signalling and extracellular proteolysis. Nat. Cell Biol. 5:711-719, 2003.

${ }^{42}$ Scanlon, E. F., and S. Murthy. The process of metastasis. CA Cancer J. Clin. 41:301-305, 1991.

${ }^{43}$ Schedin, P., and P. J. Keely. Mammary gland ECM remodeling, stiffness, and mechanosignaling in normal development and tumor progression. Cold Spring Harb. Perspect. Biol. 3:a003228, 2011.

${ }^{44}$ Shieh, A. C., H. A. Rozansky, B. Hinz, and M. A. Swartz. Tumor cell invasion is promoted by interstitial flow-induced matrix priming by stromal fibroblasts. Cancer Res. 71:790-800, 2011.

${ }^{45}$ Sporn, M. B. The war on cancer. Lancet 347:1377-1381, 1996.

${ }^{46}$ Vishwanath, M., L. Ma, C. A. Otey, J. V. Jester, and W. M. Petroll. Modulation of corneal fibroblast contractility within fibrillar collagen matrices. Invest. Ophthalmol. Vis. Sci. 44:4724-4735, 2003.

${ }^{47}$ Wirtz, D., K. Konstantopoulos, and P. C. Searson. The physics of cancer: the role of physical interactions and mechanical forces in metastasis. Nat. Rev. Cancer 11:512$522,2011$.

${ }^{48}$ Wolf, K., I. Mazo, H. Leung, K. Engelke, U. H. von Andrian, E. I. Deryugina, A. Y. Strongin, E. B. Brocker, and P. Friedl. Compensation mechanism in tumor cell migration: mesenchymal-amoeboid transition after blocking of pericellular proteolysis. J. Cell Biol. 160:267-277, 2003.

${ }^{49}$ Wolf, K., Y. I. Wu, Y. Liu, J. Geiger, E. Tam, C. Overall, M. S. Stack, and P. Friedl. Multi-step pericellular proteolysis controls the transition from individual to collective cancer cell invasion. Nat. Cell Biol. 9:893-904, 2007.

${ }^{50}$ Wolf, K., S. Alexander, V. Schacht, L. M. Coussens, U. H. von Andrian, J. van Rheenen, E. Deryugina, and P. Friedl. 
Collagen-based cell migration models in vitro and in vivo. Semin. Cell Dev. Biol. 20:931-941, 2009.

${ }^{51}$ Wong, L. H., N. A. Kurniawan, H.-P. Too, and R. Rajagopalan. Spatially resolved microrheology of heterogeneous biopolymer hydrogels using covalently bound microspheres. Biomech. Model. Mechanobiol. 2013. doi: 10.1007/s10237-013-0538-4.
${ }^{52} \mathrm{Yu}, \mathrm{X}$., and L. M. Machesky. Cells assemble invadopodialike structures and invade into matrigel in a matrix metalloprotease dependent manner in the circular invasion assay. PLOS ONE 7:e30605, 2012.

${ }^{53}$ Zaman, M. H. The role of engineering approaches in analysing cancer invasion and metastasis. Nat. Rev. Cancer 13:596-603, 2013. 